Revista Arbitrada Interdisciplinaria de Ciencias de la Salud. SALUD Y VIDA

Volumen 3. Número 6. Año 3. Julio - Diciembre 2019

Hecho el depósito de Ley: FA2016000010

ISSN: $2610-8038$

FUNDACIÓN KOINONIA (F.K)

Santa Ana de Coro, Venezuela.

Génesis Jesús Reyes Pico; Carlos Andres Pesantes Quijije; Triana Lucero Jimbo Bello

DOI: http://dx.doi.org/10.35381/s.v.v3i6.338

\title{
La estética: Clave principal en la rehabilitación oral integral
}

Sesthetics: Main key in integral oral rehabilitation

\author{
Génesis Jesús Reyes Pico \\ genesis reyes24@hotmail.com \\ Universidad Laica Eloy Alfaro de Manabí \\ Ecuador \\ https://orcid.org/0000-0003-0895-0687 \\ Carlos Andres Pesantes Quijije \\ Carlosandres pq@hotmail.com \\ Universidad Laica Eloy Alfaro de Manabí \\ Ecuador \\ https://orcid.org/0000-0003-3393-1103 \\ Triana Lucero Jimbo Bello \\ trianalucero1@hotmail.com \\ Universidad Laica Eloy Alfaro de Manabí \\ Ecuador \\ https://orcid.org/0000-0001-8194-8239
}

Recibido: 2 de mayo de 2019

Aprobado: 2 de junio de 2019

\section{RESUMEN}

La búsqueda de una mejor estética dentofacial persiste en la sociedad moderna. Se ha buscado tratamiento para pacientes, modalidades para mejorar la sonrisa y la estética dento-facial. De este modo, la rehabilitación estética oral integral requiere un manejo tambien integral por parte de un equipo multidisciplinario que involucra diferentes especialidades como endodoncia, ortodoncia, prostodoncia y periodoncia. Este artículo tiene como objetivo presentar un caso clínico que describe un manejo multidisciplinario en un paciente de 52 años con restauración estética de la sonrisa

Descriptores: Restauración; Estética; Dental; Cerámica; Sonrisa. 
Revista Arbitrada Interdisciplinaria de Ciencias de la Salud. SALUD Y VIDA

Volumen 3. Número 6. Año 3. Julio - Diciembre 2019

Hecho el depósito de Ley: FA2016000010

ISSN: $2610-8038$

FUNDACIÓN KOINONIA (F.K)

Santa Ana de Coro, Venezuela.

Génesis Jesús Reyes Pico; Carlos Andres Pesantes Quijije; Triana Lucero Jimbo Bello

\begin{abstract}
The search for a better dentofacial aesthetic persists in modern society. It has sought treatment for patients, modalities to improve the smile and dento-facial aesthetics. Thus, comprehensive oral aesthetic rehabilitation also requires comprehensive management by a multidisciplinary team that involves different specialties such as endodontics, orthodontics, prosthodontics and periodontics. This article aims to present a clinical case that describes a multidisciplinary management in a 52-year-old patient with aesthetic restoration of the smile.
\end{abstract}

Descriptors: Restoration; Aesthetics; Dental; Ceramics; Smile.

\title{
INTRODUCCIÓN
}

Es vital para la protección y la integridad del sistema masticatorio, que los dentistas tengan la responsabilidad de desarrollar una forma y función adecuadas al emprender cualquier caso restaurador (McIntyre, 2000). La clave para la estabilidad a largo plazo, la guía anterior correcta o la dirección de las fuerzas aplicadas a la dentición anterior, puede tener un efecto significativo en el éxito o el fracaso de las restauraciones.

\section{Oclusión}

A menudo se pasa por alto, la oclusión juega un papel importante no solo en la función, sino también en la estética (Mclntyre, 2000). Para lograr resultados predecibles, es necesario que los dentistas y ceramistas comprendan completamente las fuerzas masticatorias que se aplicarán a las restauraciones, incluidos los hábitos parafuncionales del paciente (Mclntyre, 2000).

Los dentistas suelen trabajar con la relación oclusal de un paciente tal como existe (Alex, 2007). Sin embargo, esto a menudo resulta perjudicial para la estabilidad del sistema masticatorio y puede causar una falla temprana de las restauraciones (Alex, 2007). Manifestarse como dolor muscular, problemas en las articulaciones, desgaste, astillado, movilidad dental, sensibilidad, una mordida incómoda y una variedad de otros síntomas, la maloclusión puede ser la causa de muchos problemas para los pacientes 
Revista Arbitrada Interdisciplinaria de Ciencias de la Salud. SALUD Y VIDA

Volumen 3. Número 6. Año 3. Julio - Diciembre 2019

Hecho el depósito de Ley: FA2016000010

ISSN: $2610-8038$

FUNDACIÓN KOINONIA (F.K)

Santa Ana de Coro, Venezuela.

Génesis Jesús Reyes Pico; Carlos Andres Pesantes Quijije; Triana Lucero Jimbo Bello

(Alex, 2007). Si no se aborda durante el proceso restaurador estético y funcional, fracturas de porcelana, fatiga del cemento y lavado de los agentes adhesivos (lo que lleva a caries secundaria) puede esperarse (Alex, 2007; Kinsel y Lin, 2009).

\section{Orientación anterior}

Proporcionado por los contornos linguales de los 6 dientes anteriores maxilares cuando entran en contacto con la cara facial de los 8 dientes mandibulares en oclusión céntrica y movimientos protrusivos y extrusivos, la guía anterior protege a los dientes posteriores de una fuerza lateral excesiva. las fuerzas laterales deben dirigirse hacia los dientes anteriores (Sorensen et al., 1999). Como resultado de la longitud de la raíz y la posición con respecto a la articulación temporomandibular (ATM), los caninos proporcionan la mayor cantidad de reducción de fuerza a la dentición posterior porque dirigen un patrón masticatorio vertical (Sorensen et al., 1999). La oclusión protegida canina minimiza el riesgo del paciente de desarrollar problemas de trastorno temporomandibular, ya que hay una reducción en los contactos laterales e interferentes de los dientes.

Para lograr el éxito de la restauración estética, los dentistas y su equipo de laboratorio dental deben controlar las fuerzas aplicadas a los dientes (Sorensen et al., 1999). En la dentición natural, los dientes anteriores excluyen (separan) los dientes posteriores en todos los movimientos de la mandíbula para evitar fuerzas destructivas horizontales y laterales. en los dientes posteriores (Sorensen et al., 1999). En general, crear armonía dentro de los contornos linguales de los dientes anteriores superiores y el sistema masticatorio es el factor más importante en la salud y la estabilidad de la oclusión y el tratamiento restaurador del paciente (Sorensen et al., 1999).

\section{Dimensión vertical}

La dimensión vertical oclusal (DVO) (también conocida como la dimensión vertical de la oclusión) es la distancia entre cualquier punto del maxilar y cualquier punto de la mandíbula donde los dientes están en máxima intercuspidación. Se establece durante 
Revista Arbitrada Interdisciplinaria de Ciencias de la Salud. SALUD Y VIDA

Volumen 3. Número 6. Año 3. Julio - Diciembre 2019

Hecho el depósito de Ley: FA2016000010

ISSN: $2610-8038$

FUNDACIÓN KOINONIA (F.K)

Santa Ana de Coro, Venezuela.

Génesis Jesús Reyes Pico; Carlos Andres Pesantes Quijije; Triana Lucero Jimbo Bello

el crecimiento y el desarrollo de los dientes (Glosario de términos prostodónticos, 1999). En el contexto clínico, la DVO a menudo se modifica para corregir problemas de visualización de dientes y encías, mejorar las relaciones oclusales y ganar espacio al restaurar dientes cortos o desgastados (Spear, 2007). Sin embargo, es necesario tener precaución. , cuando se selecciona un nuevo DVO, ya que pueden producirse efectos negativos para la ATM, el habla y la estabilidad. Posición, las necesidades restaurativas de la dentición anterior y posterior, y las necesidades funcionales de la sobremordida y la sobrejet (Spear, 2007).

\section{Bruxing}

De los muchos hábitos parafuncionales con los que los pacientes pueden presentarse, el bruxing es comúnmente una causa de desgaste y dolor severos (Mclntyre, 2000). Considerado típicamente como una disfunción de la ATM, el bruxing es en realidad el resultado de una disfunción neurológica y también puede ser una función liberadora de estrés (Mclntyre, 2000). No importa el caso, el dentista y el equipo del laboratorio dental deben hacer consideraciones cuidadosas al tratar a pacientes con bruxismo (McIntyre, 2000). No se deben usar materiales propensos a astillarse y fracturarse, ya que el hábito de bruxismo del paciente probablemente continuará incluso después del tratamiento restaurador. En los casos que requieren restauraciones totalmente cerámicas, se requiere un sistema que sea resistente al desgaste y a las fracturas para satisfacer las necesidades del paciente bruxer y evitar la necesidad de protectores bucales protectores (McIntyre, 2000; Alex, 2007; Kinsel y Lin, 2009).

Históricamente, los sistemas cerámicos convencionales han proporcionado a los profesionales dentales tratamientos predecibles y eficientes para casos altamente estéticos (Guess et al, 2010). Sin embargo, la mayoría de los materiales estéticos a menudo fallan cuando se los somete al estrés de los hábitos parafuncionales típicos (Guess et al, 2010). La elección de dentistas y técnicos dentales para muchos años, las restauraciones de cerámica a base de metal resolvieron algunos de los problemas de 
fractura y astillamiento asociados con los sistemas de porcelana convencionales (Kinsel y Lin, 2009; Guess et al., 2010). Aunque estos sistemas podían soportar las tensiones de la función, a menudo carecían de la estética de todas las cerámicas, y todavía se producían algunas astillas (Kinsel y Lin, 2009; Guess et al., 2010).. Utilizando una técnica monolítica, los sistemas cerámicos presionables de disilicato de litio (como IPS e.max Press e IPS e.max CAD [Ivoclar Vivadent]) ayudan a resolver algunos de estos problemas históricos, al tiempo que exhiben las características físicas y ópticas deseadas por incluso el más exigente de pacientes y profesionales dentales.

La cerámica de vidrio de disilicato de litio, que ofrece el ajuste, la forma y la función de las cerámicas prensibles convencionales, también demuestra una mayor resistencia y propiedades ópticas optimizadas. Puentes anteriores de unidades, puentes premolares de 3 unidades, coronas primarias de telescopios y superestructuras de implantes, el disilicato de litio ofrece soluciones para muchas necesidades clínicas diferentes (Sorensen et al., 1999; Höland et al., 2000; Kheradmandan et al., 2001). El disilicato de litio se puede usar en entornos que requieren cementación convencional (cuando la resistencia y la forma de retención son adecuadas posible en el diseño de la preparación) o la unión de resina adhesiva, y las propiedades estéticas y físicas de este material permiten a los dentistas y ceramistas abordar las preocupaciones funcionales y estéticas de una manera predecible.

\section{REPORTE DE UN CASO}

\section{Diagnóstico y planificación del tratamiento}

Una mujer de 52 años se mostró preocupada por su mordida, sus hábitos de molienda, su presentación gingival y la falta general de estética en su dentición. Tras consultar con el paciente, se descubrió que a medida que envejecía, notó que sus dientes se veían gradualmente peor. Ella dijo que nunca le había gustado realmente la apariencia de sus dientes, y que su situación actual afectó negativamente su confianza en sí misma. Quería usar lápiz labial, algo que nunca había hecho por miedo a llamar la 
atención sobre su sonrisa. Como madre y miembro activo de su comunidad, quería una mejor sonrisa.

Tras el examen clínico, se observó un desgaste severo causado por bruxing. Este hábito parafuncional condujo a problemas con la proporción, longitud, contorno, color y función del diente. Además, los niveles gingivales del paciente eran desiguales y había una visualización gingival excesiva (antes de la imagen y las figuras 1 y 2 ).
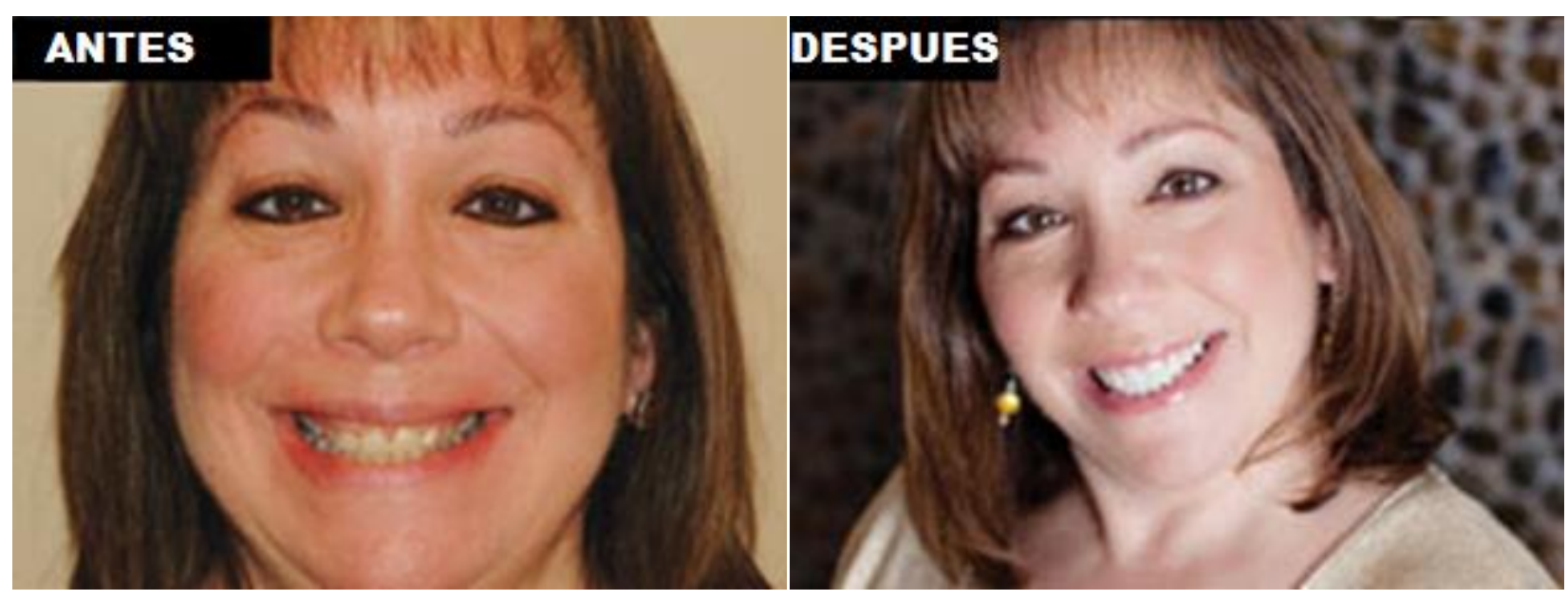

Imagen antes. Retrato preoperatorio de la ceja del Imagen después. Los resultados finales se ven paciente al área de la barbilla. mejor en esta foto: un aspecto absolutamente elegante.
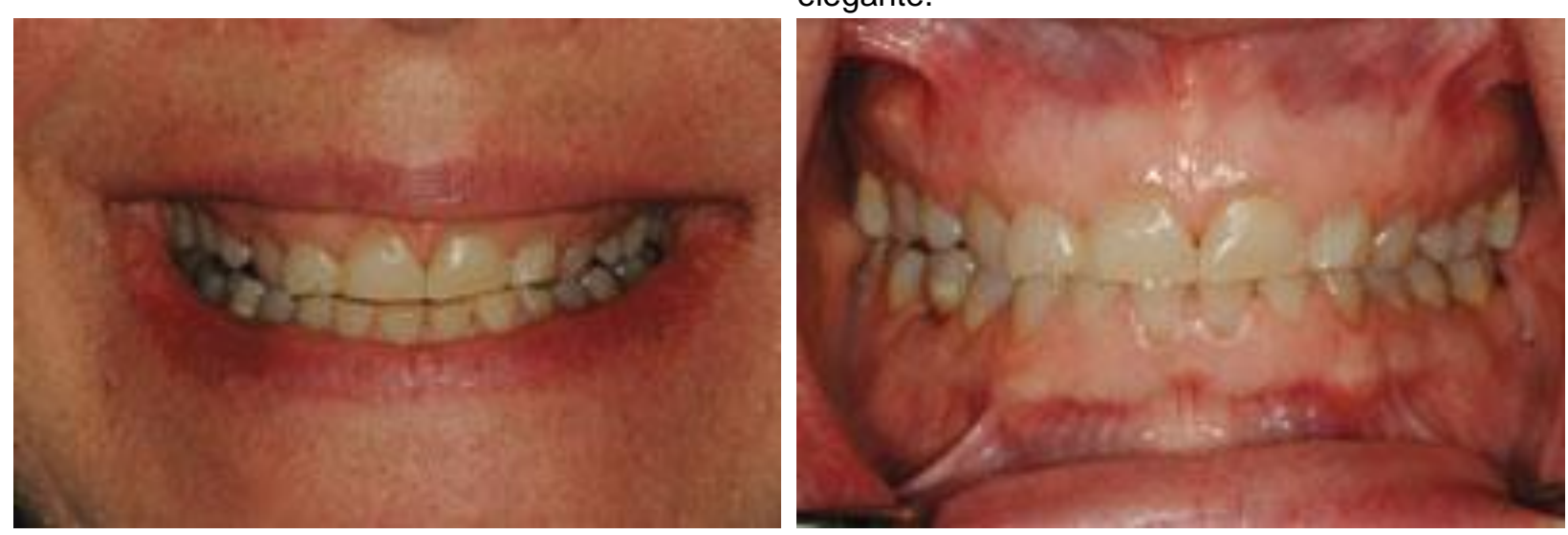

Figura 1. Fotografía preoperatoria de la sonrisa del Figura 2. Vista retraída preoperatoria de la dentición paciente que muestra problemas estéticos. del paciente.

La paciente era muy consciente de sus problemas dentales y de que necesitaría una reconstrucción completa para corregirlos. Debido a que el paciente era un bruxer 
severo, se necesitaría un material restaurador que no se fracturara o astillara fácilmente. Primero, sería necesario abordar el plano oclusal del paciente y los desafíos de orientación anterior, al tiempo que se eliminan las interferencias posteriores. Se planificó un encerado de diagnóstico para encontrar una solución y guiar al equipo dental en la corrección de problemas estéticos y oclusales. Además, se utilizaría una gingivectomía para lograr los niveles gingivales adecuados y estéticos.

Debido a que a la paciente le faltaban sus primeros premolares, los 16 dientes anteriores debían restaurarse primero, en lugar de los 20 típicos. Los primeros y segundos molares inicialmente quedarían fuera de la oclusión. Además, se recomendó al paciente que los terceros molares restantes se extraigan al menos 2 meses antes de iniciar el tratamiento. Se prefirió el recubrimiento de todos los dientes mandibulares, en lugar de usar coronas, para evitar la preparación lingual de los incisivos inferiores ya que ya eran delgados.

También se incluyeron en el plan de tratamiento las carillas para dientes Nos. 6 a 11 y carillas para los premolares superiores. Para lograr el DVO postoperatorio deseado, las superficies oclusales de los premolares debían estar completamente cubiertas. Sin embargo, no fue necesario colocar el margen lingual de las preparaciones más de la mitad de la pared palatina del diente, ya que las restauraciones se unirían adhesivamente en su lugar.

\section{Selección de material}

La paciente quería que sus restauraciones imitaran la apariencia de los dientes naturales. En este caso, elegimos utilizar un disilicato de litio prensado totalmente cerámico (IPS e.max Press) para ayudarnos a cumplir el objetivo estético declarado del paciente. Cuando se usa como material monolítico (sin el uso de capas de porcelanas), IPS e.max Press es un material totalmente cerámico muy resistente (resistencia a la flexión biaxial de $400 \mathrm{MPa}$ ) que se puede unir con resina para obtener la máxima resistencia. Si lo desea, también se puede presionar para obtener un contorno 
completo, y luego recortarlo y crear capas para crear cualquier efecto especial que pueda ser necesario dentro de la zona estética. Demostrando una resistencia excepcional, junto con altos valores estéticos y propiedades ópticas, el disilicato de litio es ideal para casos restaurativos donde la estética y la función deben ser predeciblemente entregadas.

\section{Diagnóstico clínico}

Primero se completó una evaluación exhaustiva de TMJ utilizando un análisis de vibración articular (Bio-JAV Scan [Bio-RESEARCH Associates]) para evaluar la función y los patrones de vibración de la TMJ. Todo el trabajo periodontal y restaurativo anterior se trazó. La historia dental del paciente no era notable, sin trabajo periodontal o dental importante. Además, su TMJ se encontró estable antes del tratamiento.

Luego se tomó un conjunto completo de fotografías y radiografías de diagnóstico, y se realizaron impresiones superiores e inferiores utilizando un material de impresión de polisiloxano de vinilo (PSV) (Impresión 3 [3M ESPE]) para permitir múltiples vertidos para la fabricación de modelos de diagnóstico. Estas impresiones también le permitieron al paciente revisar sus restauraciones iniciales y finales en el articulador (Stratos [Ivoclar Vivadent]).

Utilizando un desprogramador anterior que el paciente usó durante varios días, se tomó un registro de mordida de oclusión céntrica, y se estimó su nueva DVO posrestaurativa. Al usar el desprogramador, se capturó fácilmente una posición condilar repetible durante el proceso de registro de la mordida. Además, al usar la unión de cemento y esmalte del incisivo superior e inferior (CEJ) a las mediciones de CEJ usando calibradores digitales (Erskine Dental), se establecieron aún más las dimensiones verticales adecuadas. Simple y preciso, este método proporcionó al equipo del laboratorio dental información suficiente para montar el caso en relación céntrica con el DVO correcto (estimado después de la restauración). Con fotografías clínicas, el ceramista pudo encerar el estuche a la forma y función ideales. 
Como se mencionó anteriormente, la arquitectura gingival también fue muy importante para lograr la estética final deseada de este caso. El sondeo de los dientes anteriores y el hueso mostró que no sería necesario alargar la corona ósea. Ahorrando tiempo y dinero para el paciente, se determinó que a pesar de la cantidad excesiva de tejido gingival facial que requería extirpación, no había problemas con el ancho biológico. Trabajando con el ceramista, se mapearon los cambios de tejido propuestos para el día de la preparación y se creó una guía de reducción de tejido.

\section{Protocolo de preparación clínica}

El paciente fue anestesiado con una solución de hidrocloruro de articaína al 4\% con epinefrina 1: 100,000 (Septocaína [Septodont]) usando un sistema de anestesia computarizado (The Wand [CompuDent]) para proporcionar una inyección más cómoda. Mientras el paciente esperaba que la anestesia surta efecto, una matriz de masilla dura (Sil-Tech [Ivoclar Vivadent]) fabricada a partir del encerado de diagnóstico se llenó con un material provisional compuesto de bis-acrilo (Luxatemp [DMG America]) y sentado sobre los dientes no preparados. Como la técnica de restauración era aditiva, se eliminó muy poca estructura dental antes de este paso. Sin embargo, para asentar la matriz, se utilizó un stent de reducción inicial (Figura 3). Luego se retiró la matriz y se usó como guía para evitar la preparación excesiva de los dientes (Figura 4).

La guía de tejido realizada durante la etapa de diagnóstico se usó para marcar las reducciones de tejido necesarias que debían realizarse antes de cualquier preparación dental. Para realizar la gingivectomía, se utilizó una unidad de radiocirugía (Ellman International) de acuerdo con el stent de reducción. Luego se extrajo la guía y se retiró el exceso de tejido donde fue necesario para establecer una vieira uniforme en cada diente.

La preparación del diente se inició con fresas de diamante (KOMET USA) y una pieza de mano ELECTROtorque (KaVo). Utilizando discos de pulido (Sof-Lex [3M ESPE]), se eliminaron los bordes ásperos / afilados de las preparaciones, y se les dio un pulido 
final. Inmediatamente después, las impresiones finales se realizaron utilizando bandejas de impresión (Bandejas de flujo dirigido [3M ESPE]) llenas de un material de impresión PSV de cuerpo pesado (Impresión 3). Para garantizar impresiones exitosas, los preparativos se mantuvieron limpios y sin líquidos durante todo el proceso.

Para mantener la DVO propuesta correcta durante todo el proceso de tratamiento, se creó una "plantilla de mordida" a partir de un material rígido de registro de mordida PSV en el encerado articulado de los molares. Los 2 registros, derecho e izquierdo, se usaron durante todo el tratamiento para la verificación de la mordida y para mantener la dimensión vertical correcta. Esta "plantilla de mordida" también ayudó al ceramista de laboratorio a montar los modelos de preparación superior e inferior en la dimensión vertical correcta.
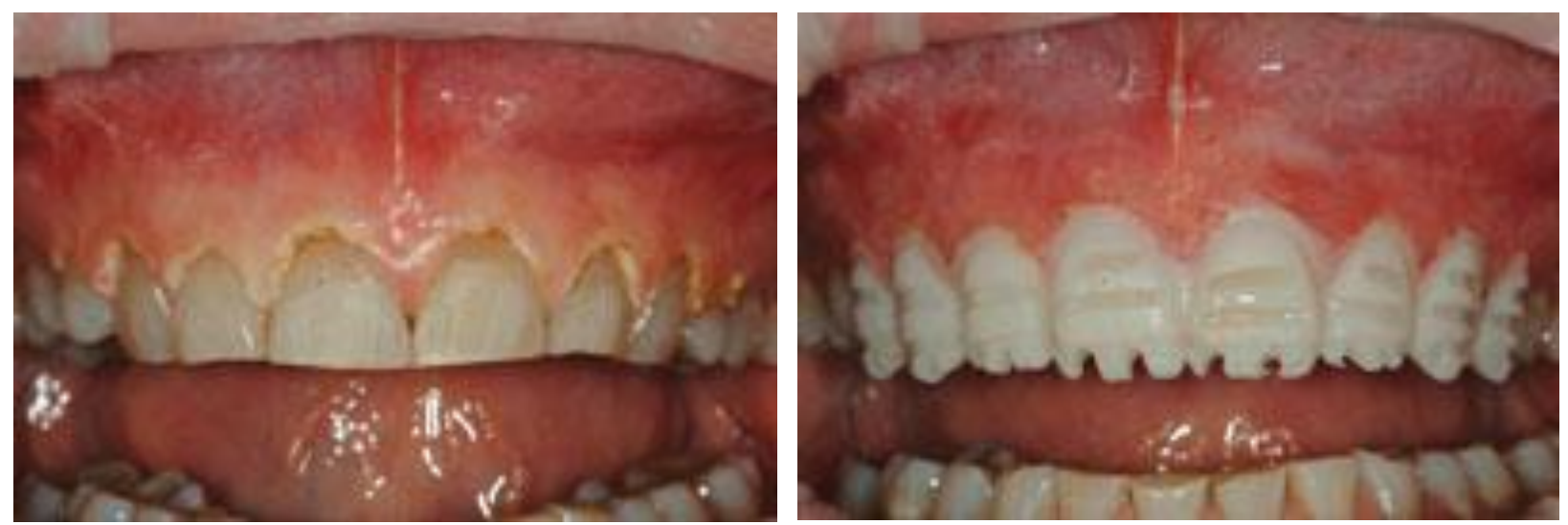

Figura 3. Después de la gingivectomía, se usó una Figura 4. Usando una fresa de corte de $0,7 \mathrm{~mm}$ de fresa de diamante en las superficies faciales de los profundidad (KOMET USA), el clínico se preparó en dientes para garantizar que la matriz se asentaría la maqueta, lo que ayudó a preservar la mayor completamente y la maqueta de los provisionales cantidad posible de estructura dental facial. permanecería en su lugar durante las preparaciones iniciales. 

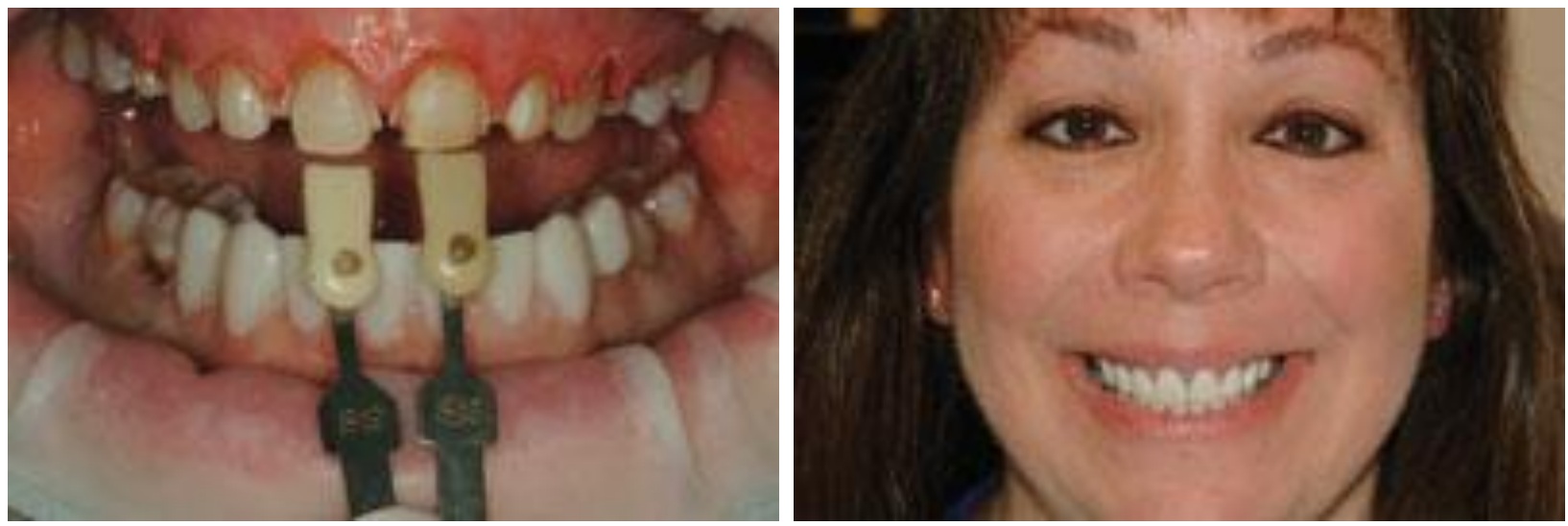

Figura 5. Después de los preparativos, se hicieron Figura 6. Se tomó una fotografía del paciente en las impresiones oclusales y se tomó una fotografía de provisionales, sin los efectos de la anestesia, lo cual sombra de tocón para comunicar la sombra general es muy importante y a menudo pasada por alto para deseada al equipo de laboratorio.

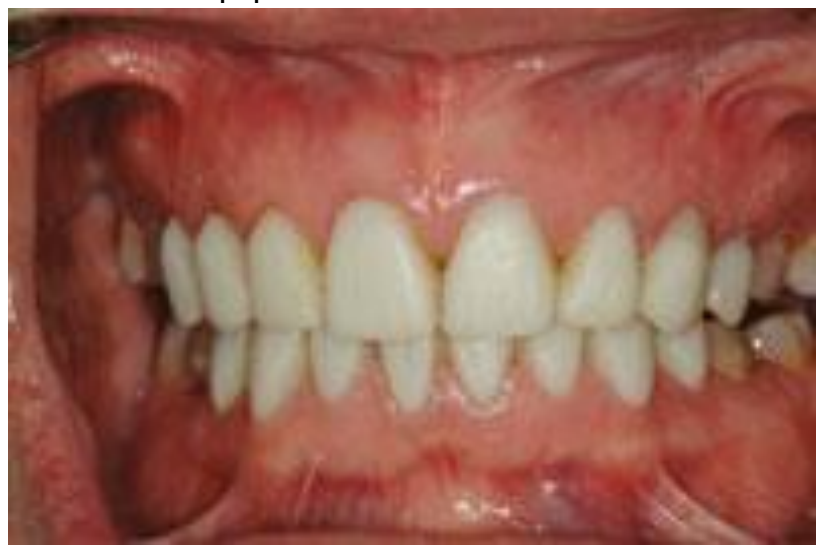

Figura 7. Vista retraída de las provisionales en su Fid lugar. el equipo de laboratorio.

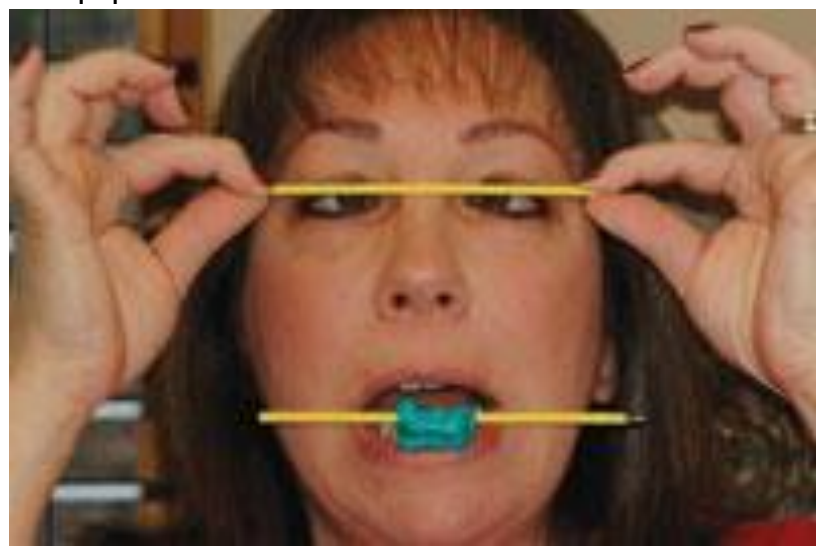

Figura 8. Fotografía de la referencia de la guía del plano horizontal. El paciente puede sostener un palo a través del puente de la nariz horizontalmente, proporcionando una referencia paralela al palo guía mientras se fija el material de registro de mordida.

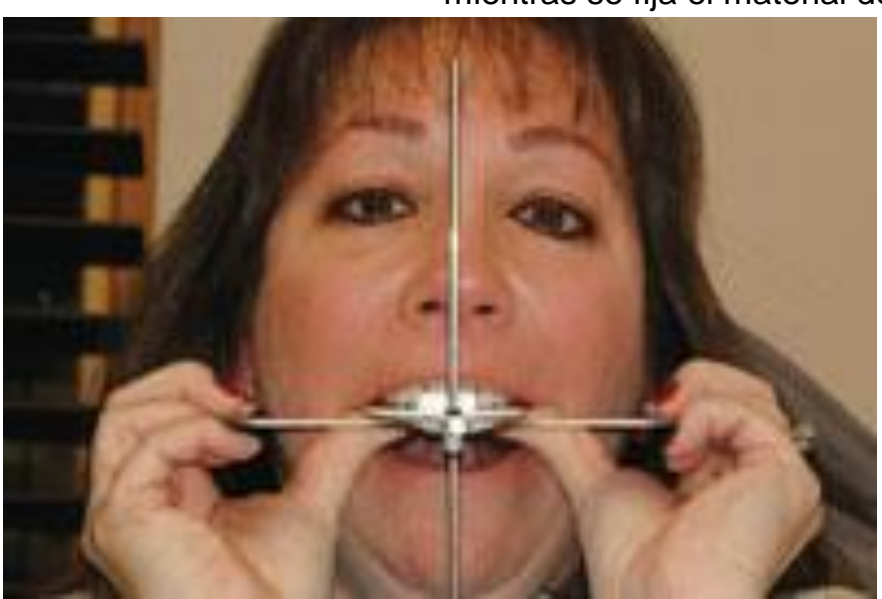


Revista Arbitrada Interdisciplinaria de Ciencias de la Salud. SALUD Y VIDA

Volumen 3. Número 6. Año 3. Julio - Diciembre 2019

Hecho el depósito de Ley: FA2016000010

ISSN: $2610-8038$

FUNDACIÓN KOINONIA (F.K)

Santa Ana de Coro, Venezuela.

Génesis Jesús Reyes Pico; Carlos Andres Pesantes Quijije; Triana Lucero Jimbo Bello

Figura 9. También se tomó un registro de arco facial (Kois Earless Facebow [Panadent]) para montar los modelos en el articulador.

Luego se tomaron sombras de tocón (guía de sombra de tocón de cromoscopio [Ivoclar Vivadent]), junto con una serie de fotografías digitales para la comunicación de los detalles del caso con el equipo del laboratorio dental (Figura 5). Una vez completado, se crearon restauraciones provisionales. Se aplicó Telio Desensitizador (Ivoclar Vivadent) a las preparaciones durante 20 segundos cada una y se diluyó al aire antes de la aplicación de OptiBond FL Primer (Kerr) en los márgenes de la preparación. Esta técnica, cuando se realiza en las preparaciones previas a la fabricación provisional, prácticamente elimina la sensibilidad y minimiza sustancialmente la microfiltración bajo las provisionales.

La matriz se cubrió con un material provisional (Luxatemp) y se colocó sobre las preparaciones. Una vez que el material provisional se había fraguado, la matriz se retiró y las provisionales se pulieron con copas y puntos de pulido (Astropol [Ivoclar Vivadent]). Se colocó un sellador y esmalte líquido de baja viscosidad (BisCover [Bisco]) para evitar manchas en las restauraciones temporales. (La clave es la tinción y no la sensibilidad. El esmalte no tiene nada que ver con la sensibilidad).

Después de que las provisionales estuvieran en su lugar, se alentó a la paciente a que tomara nota de cualquier posible cambio en la estética y la función que considerara necesario discutir.

Se tomaron fotografías en la cita posterior a la preparación y se crearon modelos de las provisionales (Figuras 6 y 7 ). El paciente hizo algunas sugerencias y se sometió a otra mordida de palo y un nuevo arco facial para evitar que las restauraciones aparezcan después del asiento final (Figuras 8 y 9). Una vez que el paciente estuvo satisfecho con las provisionales, todas las fotografías y la información diagnóstica pertinente se enviaron al laboratorio con una receta para restauraciones de disilicato de litio IPS e.max Press utilizando la técnica de corte y estratificación en todos los dientes. 


\section{Protocolo de laboratorio}

Después de recibir fotografías, información de diagnóstico y la receta del dentista, el ceramista primero vertió las impresiones en piedra. Luego se inyectó cera a través de la matriz de provisionales en los troqueles maestros (Figura 10). Los márgenes fueron sellados, y la forma y la función se desarrollaron en la cera. Una vez que esto se completó, las restauraciones estaban listas para invertir y presionar con los lingotes de disilicato de litio seleccionados (Figura 11). Los caninos y los premolares se presionaron con IPS e.max HTBL2, color blanqueador, de bajo valor, y los incisivos de IPS e.max HTBL1, de mayor valor, tanto para la parte superior como para la inferior (Figura 12). Las restauraciones inferiores también se formaron a través de la inyección de cera de la matriz provisional y se prepararon para invertir y prensar (Figura 13).

Después de que las restauraciones habían sido invertidas y luego ajustadas al modelo de trabajo, fueron examinadas contra el modelo de las provisionales (Figura 14). Se agregaron efectos de polvo internos a la reducción facial incisal teñida de 0,5 mm. Se utilizó polvo de dentina blanca opal (OE 4) para crear los lóbulos internos, mientras que el mamelón claro (MM) creó el borde incisal y un efecto halo natural (Figuras 15 y 16). Se agregaron polvos de esmalte segmentados de bajo valor y alto ópalo (OE1) (solo 0.5 $\mathrm{mm}$ ) al contorno completo en los polos incisal-mesial e incisal-distal más externos, alternando con polvos de alto valor contrastante (TI1) para imitar cualidades ópticas naturales (Figuras 17 y 18)
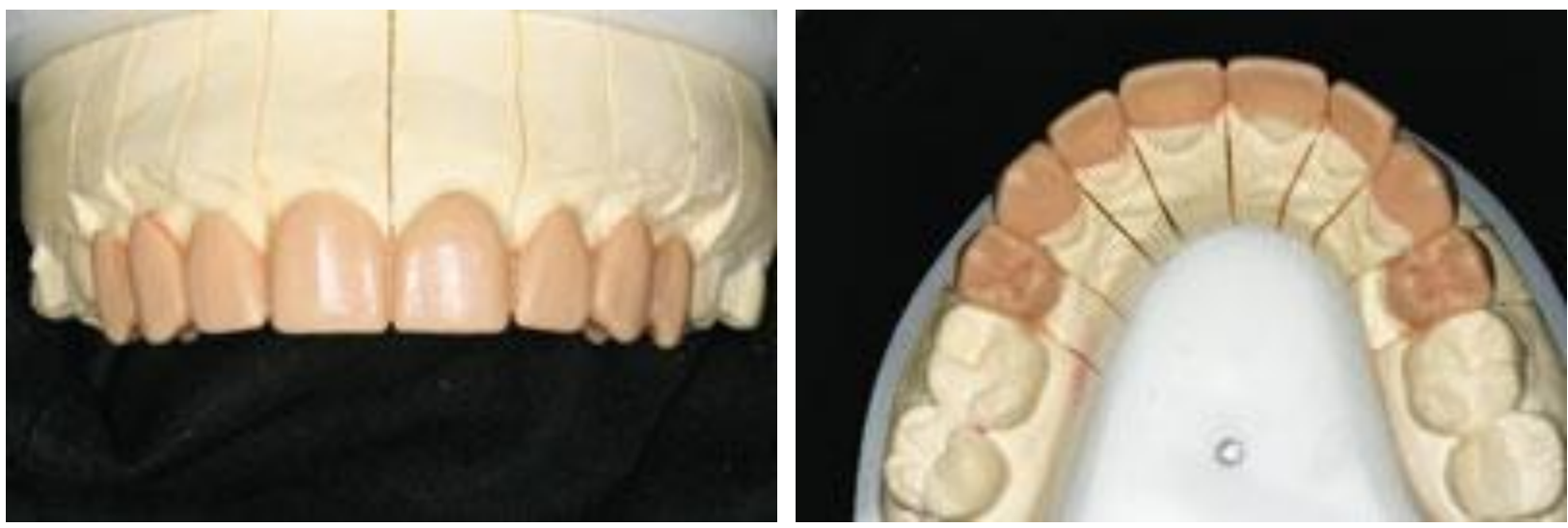

Figura 10. La cera se inyectó a través de una matriz Figura 11. Después de sellar los márgenes, la 
hecha de las provisionales modificadas y aprobadas forma y la función se desarrollaron en la cera y se

\section{en los troqueles maestros.}

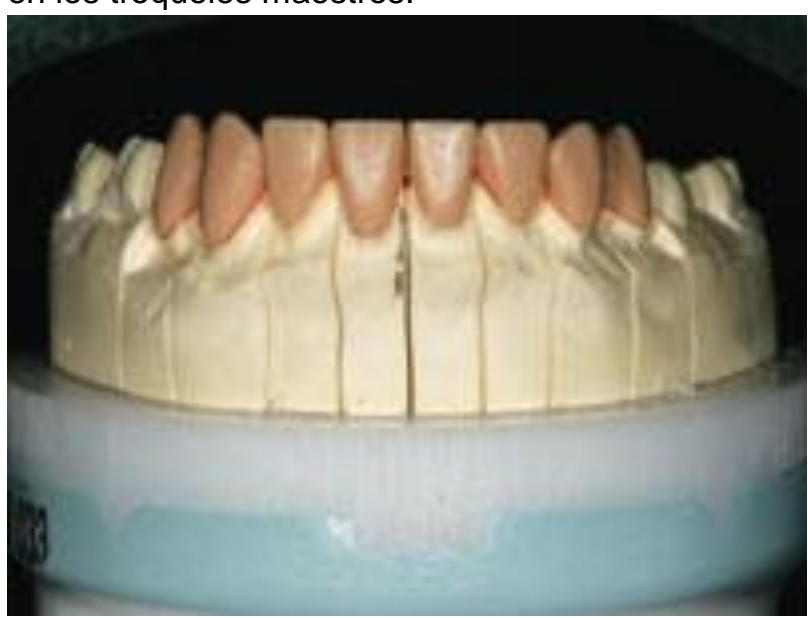

\section{prepararon para invertir y presionar.}

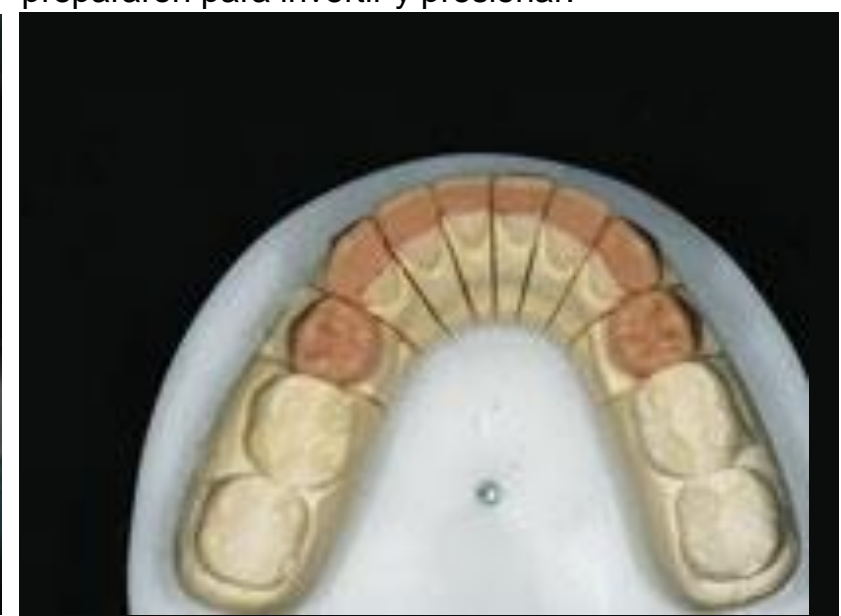

Figura 12. Los caninos y premolares se prensaron Figura 13. El encerado más bajo también se de disilicato de litio (IPS e.max Press [Ivoclar preparó para invertir y presionar. Vivadent]) y los incisivos de (IPS e.max Press).
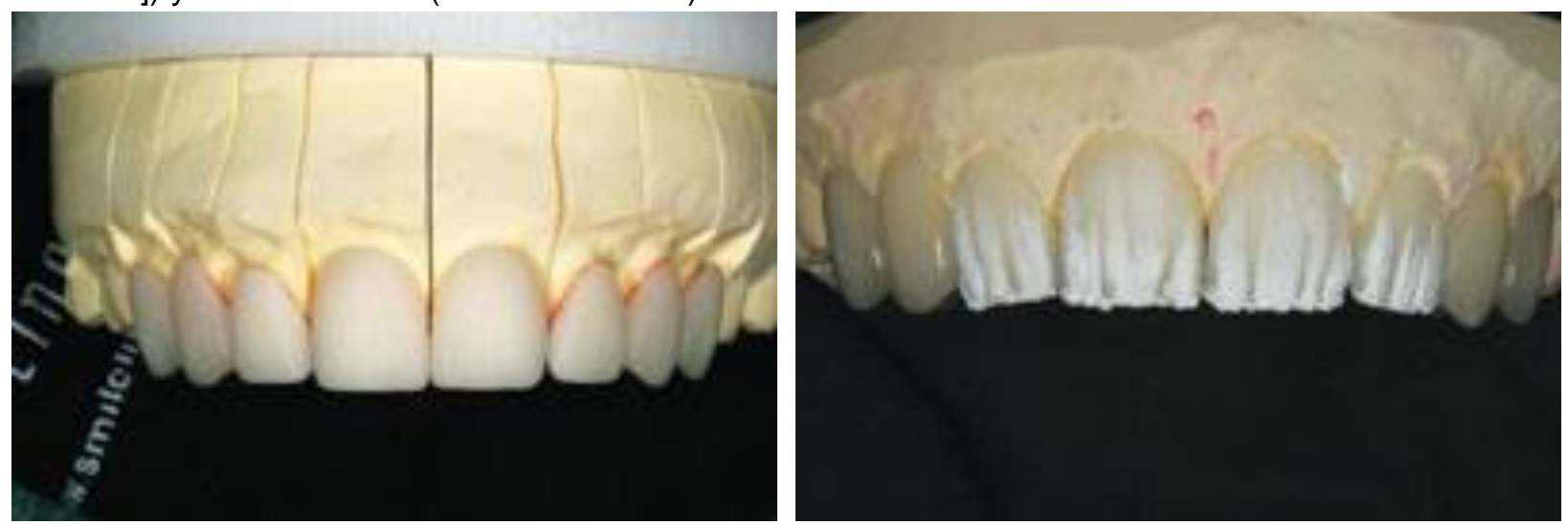

Figura 14. Después de la investigación y el ajuste, Figura 15. Los efectos internos del polvo se las restauraciones se examinaron contra el modelo agregaron a un incisal de $0,5 \mathrm{~mm}$ teñido de alto y de las provisionales.

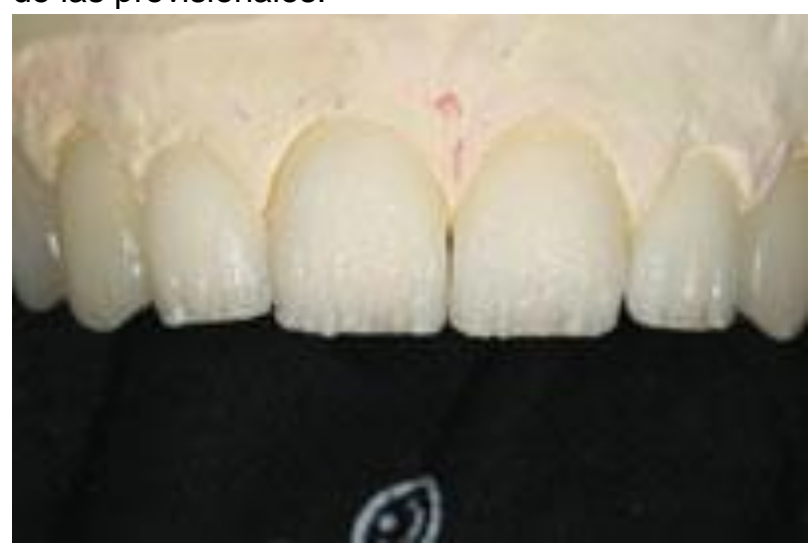
bajo valor: reducción facial.

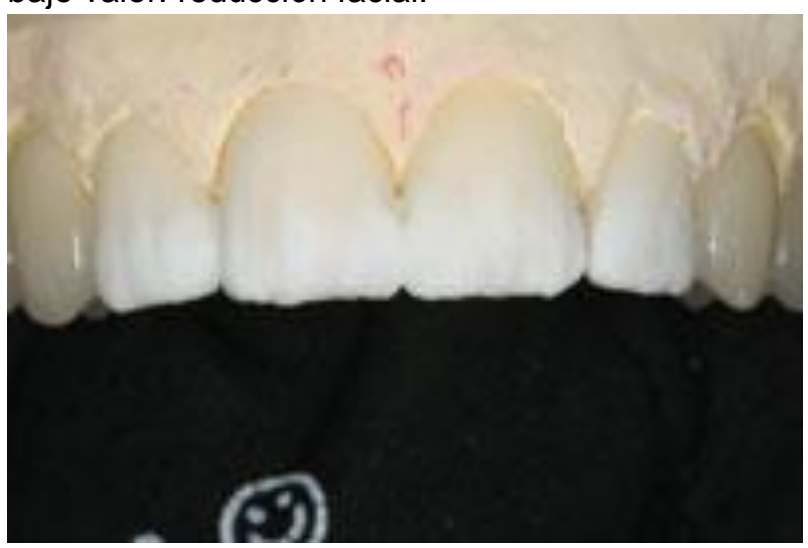


Figura 16. Vista de los efectos internos después del Figura 17. Se agregaron $0,5 \mathrm{~mm}$ de esmalte en disparo.

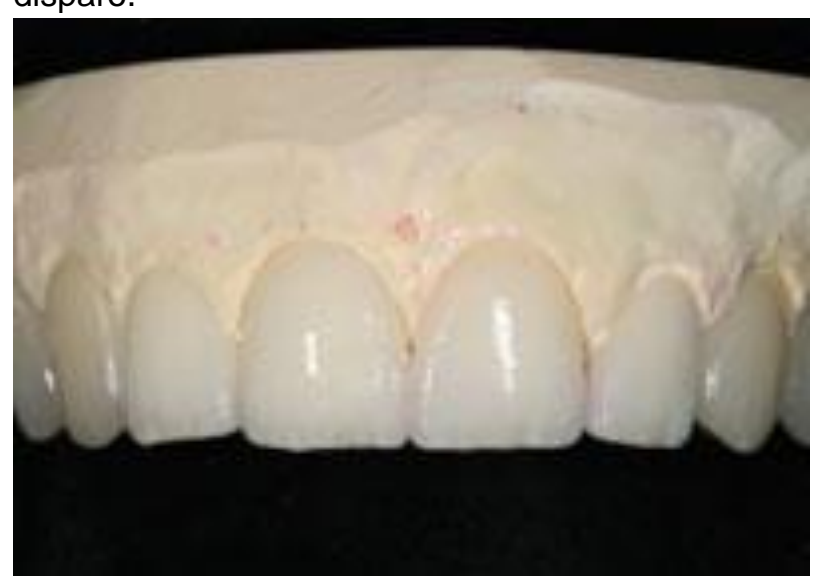
polvo al contorno completo.

Figura 18. Vista del esmalte cocido con esmalte Figura 19. Usando un lápiz rojo a base de cera, los medio para ver los efectos internos de la cerámica ángulos de línea deseados se dibujaron en las que estaban listos para dar forma y contornear.
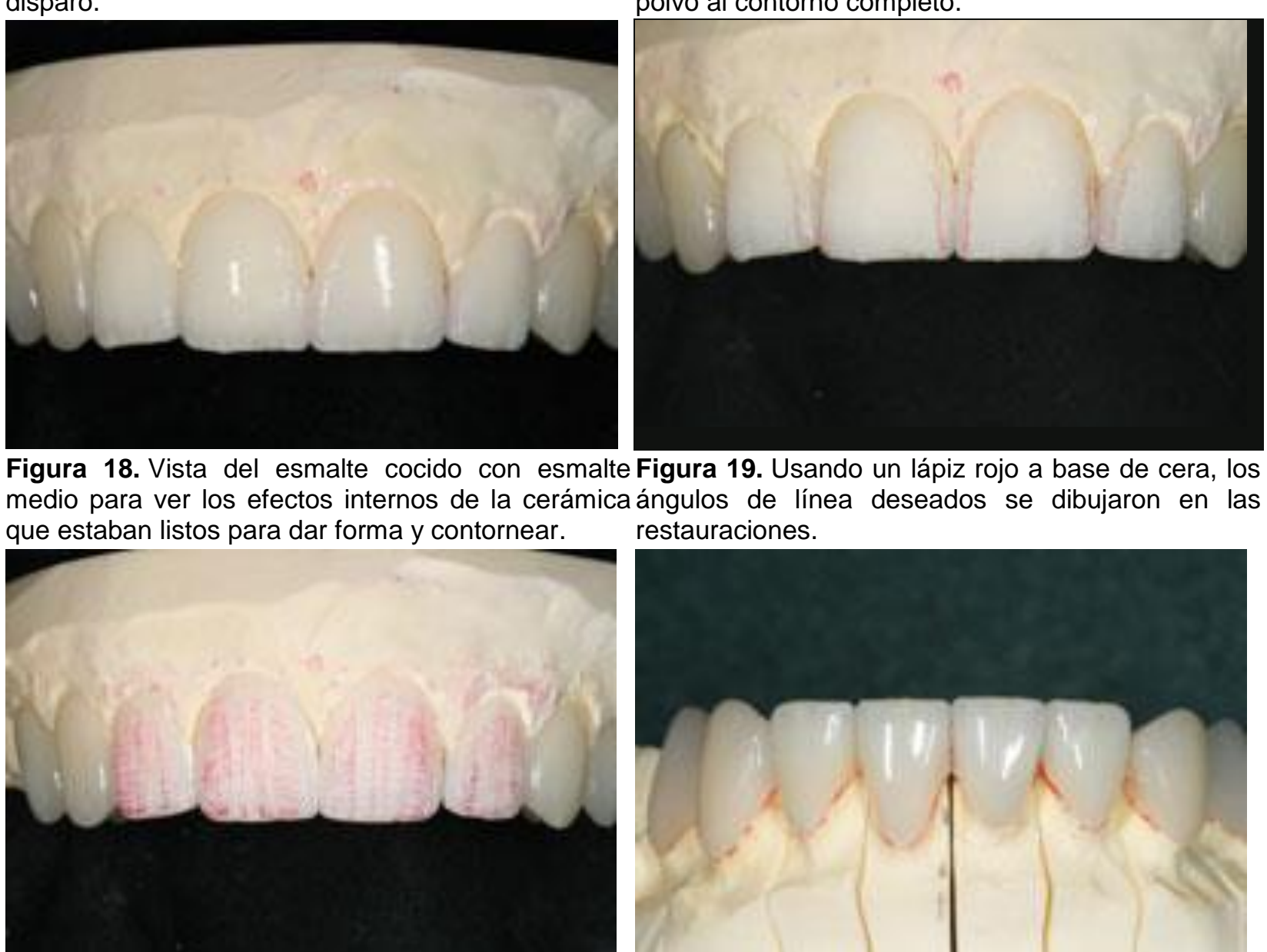
restauraciones.

Figura 20. Los lóbulos faciales se desarrollaron Figura 21. Vista de las restauraciones terminadas antes de colocar los perikymata, todo utilizando una inferiores.

fresa de diamante 842R (KOMET USA) para finalizar la superficie antes del glaseado. 


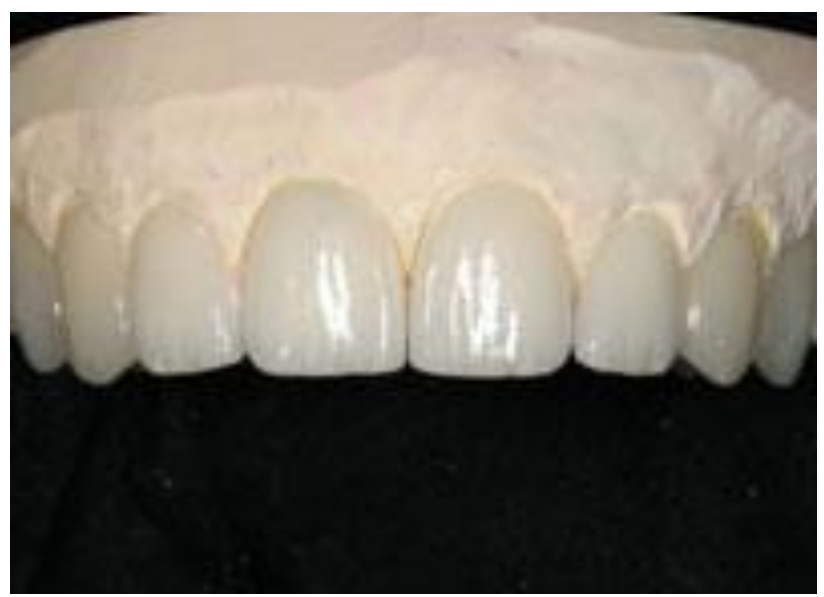

Figura 22. Vista de las restauraciones superiores terminadas.
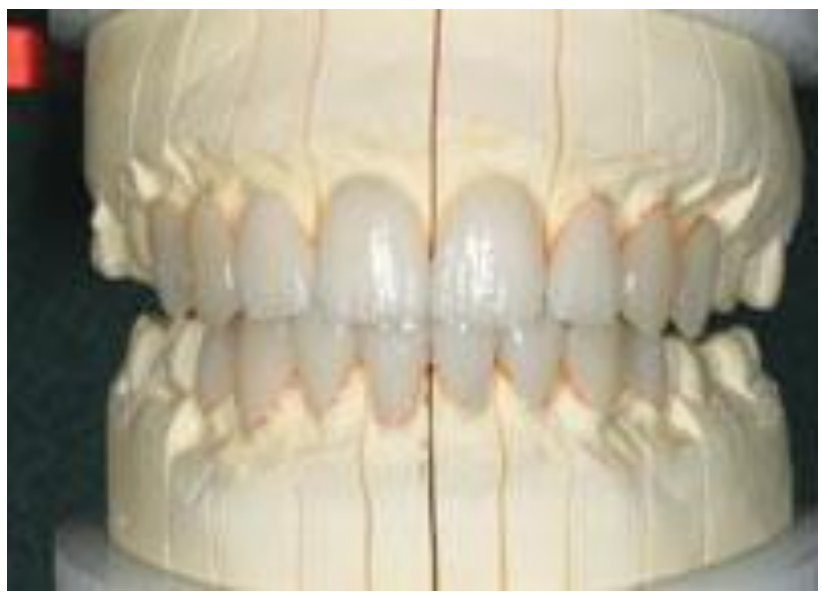

23. Vista articulada de las restauraciones finales.

Luego se usó un lápiz rojo a base de cera para dibujar los ángulos de línea deseados en las restauraciones, después de lo cual se desarrollaron las zonas de desviación interproximal utilizando la superficie plana de una rueda de goma a base de diamante (94002C [KOMET USA]) (Figura 19). Los lóbulos faciales se desarrollaron antes de impartir los perikymata, cada uno de los cuales se estableció usando una fresa de diamante (842R [KOMET USA]) para finalizar la superficie para el acristalamiento (Figuras 20 a 23).

\section{Asientos finales}

Se retiraron los provisionales y luego las preparaciones se limpiaron con cepillos (cepillos ICB [productos Ultradent]) y un exfoliante antibacteriano de clorhexidina y peróxido de hidrógeno (Consepsis Scrub [productos Ultradent]) (Figura 24). Se colocó una barrera de retracción (Optragate [Ivoclar Vivadent]) en la boca del paciente para permitir una fotografía más fácil, la comodidad del paciente y proporcionar un campo quirúrgico seco.

Las restauraciones finales se colocaron en la boca con un gel de cemento de prueba (Variolink Veneer [Ivoclar Vivadent]) diseñado para que las carillas verifiquen los contactos, el ajuste y la oclusión. El paciente estaba satisfecho con la apariencia de las 
restauraciones después de verlas por vía intraoral en diversas condiciones de iluminación, incluida la luz solar natural.

Las restauraciones se eliminaron para una limpieza profunda: el gel de prueba y otros contaminantes posibles se eliminaron de las restauraciones de disilicato de litio utilizando un grabado de ácido fosfórico al 35\% (Ultra Etch [Ultradent Products]) aplicado durante aproximadamente 20 segundos, y luego se enjuagó con agua. Todas las restauraciones se imprimaron con una imprimación de silano de cerámica (Rely- $X$ [3M ESPE]) antes de colocarlas y se secaron al aire. Luego se aplicó un adhesivo de cuarta generación (ALL BOND 3 [BISCO Dental Products]) en las superficies internas de las restauraciones. Las restauraciones se secaron al aire y se reservaron en un recipiente a prueba de luz (Vivapad [Ivoclar Vivadent]). Es correcto recubrir las superficies internas de las restauraciones con adhesivo.
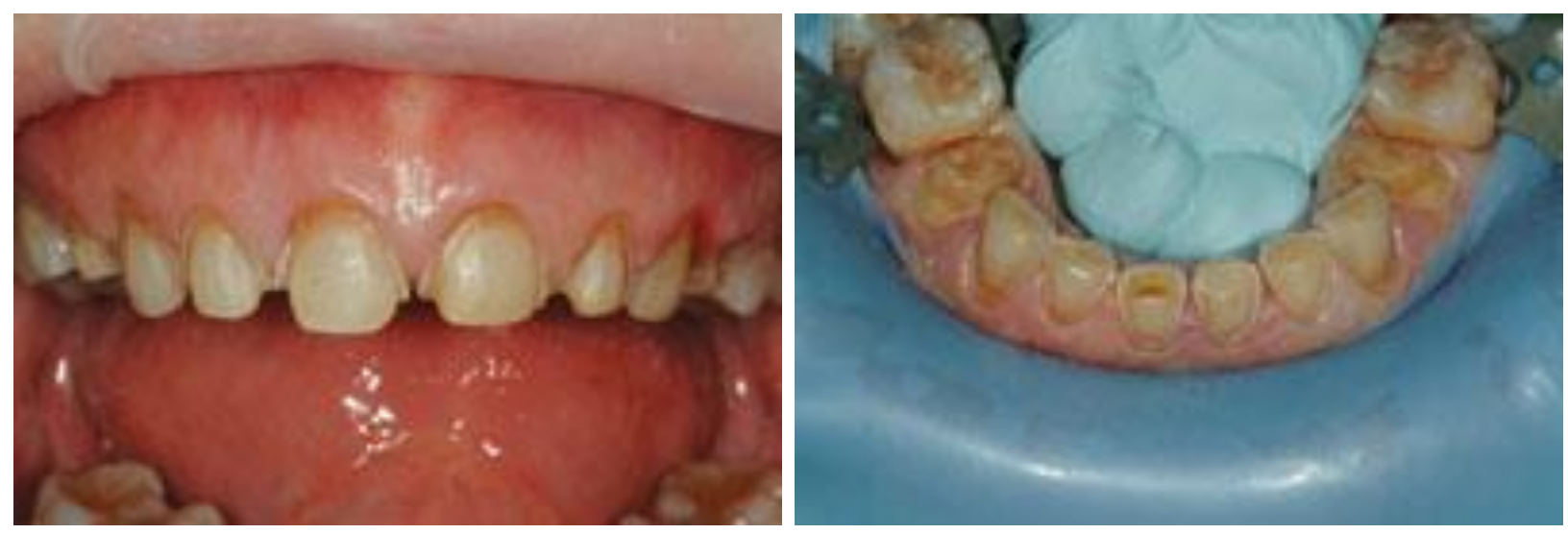

Figura 24. Se retiraron las provisionales y las Figura 25. Antes de la unión, se aplicó un agente preparaciones se limpiaron con un exfoliante desensibilizante a las preparaciones para minimizar antibacteriano de clorhexidina y peróxido de la sensibilidad postoperatoria.

hidrógeno y se aplastaron en preparación para la unión. 


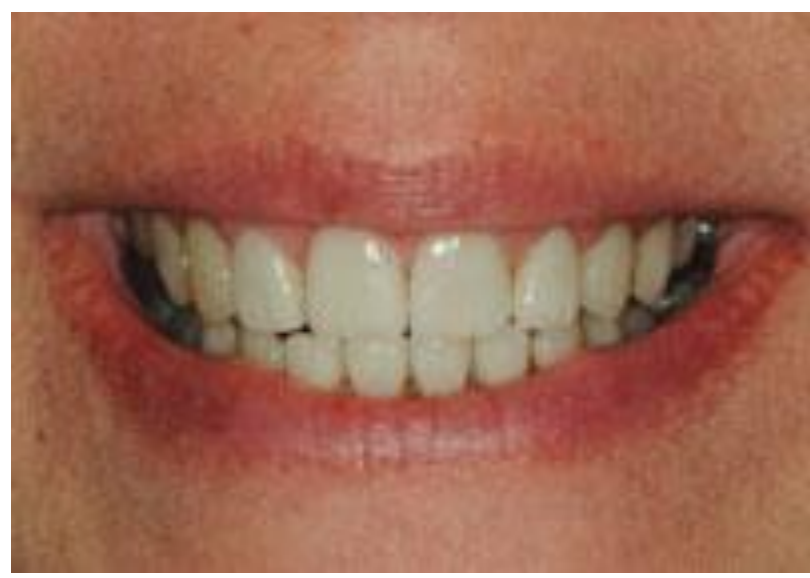

Figura 26. Vista de la sonrisa postoperatoria Figura 27. Vista postoperatoria retraída del cambio paciente.

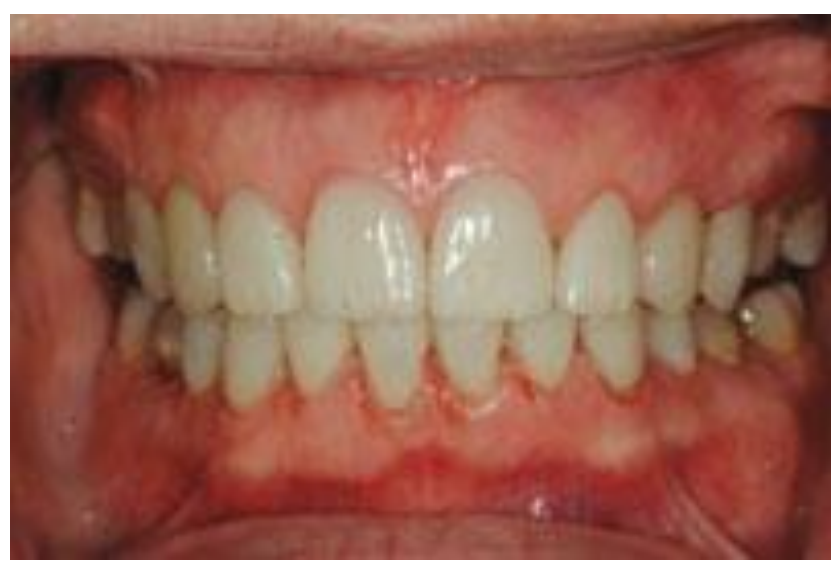
de imagen de la sonrisa del paciente.

Para garantizar una adhesión adecuada, los dientes se aislaron completamente y se grabaron con un gel de ácido fosfórico (productos Ultradent). Luego se aplicó un agente desensibilizante (Systemp.desensitizer [Ivoclar Vivadent]) durante 20 segundos cada uno, utilizando la evacuación a alta velocidad para eliminar el exceso (Figura 25). Inmediatamente después, los dientes se recubrieron con el adhesivo de cuarta generación (ALL BOND 3) durante 20 segundos cada uno, eliminando el exceso con evacuación a alta velocidad hasta que no hubo movimiento del adhesivo en ninguno de los dientes.

Luego se cargó un cemento de resina fotocurado (Variolink Veneer [Ivoclar Vivadent]) (sombra +1) en las restauraciones y se asentaron en las preparaciones. Las restauraciones se asentaron juntas y se clavaron en su lugar utilizando la punta de clavado de la luz de curado (Fase Azul [Ivoclar Vivadent]). Las restauraciones se curaron con movimientos ondulantes, facial y lingualmente, durante 2 segundos cada una. El exceso de cemento se eliminó fácilmente y los márgenes se recubrieron con grandes cantidades de gel de glicerina (lente líquida [Danville Materials]) (para evitar que se forme una capa inhibida por oxígeno) y se curaron durante 20 segundos completos en cada lado. 
Para dar a las restauraciones su ajuste final y acabado, fresas de diamante (en forma de llama: FSD4F 010, FSD4EF 010, FS4UF 010; en forma de punta: 8274 016, 274EF 016; y en forma de fútbol: 8379 023, 379EF 023 [KOMET USA] ) y se utilizó un sistema de pulido de diamantes (Optrafine [Ivoclar Vivadent]), seguido del uso de una pasta de pulido de diamantes (Diashine [VH Technologies]). Se usaron tiras de separación (KOMET USA) según fue necesario, y los contactos se volvieron a verificar para determinar el adhesivo residual, el cemento y la suavidad utilizando hilo dental. Luego se ajustó la oclusión para desarrollar una guía anterior perfecta, y no se observaron interferencias posteriores en los premolares. (Nota: los molares permanecieron fuera de la oclusión, sin embargo, porque no habían sido restaurados al momento de completar este procedimiento)

Al concluir el caso, el equipo de pacientes, dentista y laboratorio dental quedó muy satisfecho con los resultados estéticos y funcionales del tratamiento (Figuras 26 y 27 e Imagen después). La paciente estaba aún más emocionada de que pudiera usar lápiz labial por primera vez, y tener la confianza en sí misma para hacerlo sin preocuparse de que llamaría la atención sobre su sonrisa anteriormente poco estética. Para el dentista y el ceramista, el caso tuvo éxito más allá de la odontología planificada de manera predecible, sino que marcó una diferencia especial y dramática en la vida del paciente.

\section{CONCLUSIÓN}

Aunque la estética es el objetivo principal en la región anterior, los aspectos más importantes de cualquier restauración son la función y la estabilidad del sistema masticatorio (Mclntyre, 2000). Para garantizar la longevidad restaurativa, los dentistas deben considerar las fuerzas que se aplicarán a sus restauraciones y lo que afecta a la anterior la orientación, la dimensión vertical y los hábitos parafuncionales tendrán McIntyre, 2000). A partir de ahí, pueden desarrollar un plan de tratamiento para satisfacer las necesidades colectivas del paciente utilizando un material seleccionado adecuadamente. 
Revista Arbitrada Interdisciplinaria de Ciencias de la Salud. SALUD Y VIDA

Volumen 3. Número 6. Año 3. Julio - Diciembre 2019

Hecho el depósito de Ley: FA2016000010

ISSN: $2610-8038$

FUNDACIÓN KOINONIA (F.K)

Santa Ana de Coro, Venezuela.

Génesis Jesús Reyes Pico; Carlos Andres Pesantes Quijije; Triana Lucero Jimbo Bello

Si bien esto es importante para todos los casos de restauración, los autores señalan la importancia de proporcionar restauraciones predecibles, estéticas y duraderas que funcionen a largo plazo, en particular para este paciente. Es una mujer amable, cálida y activa a la que nunca le gustó su sonrisa y, como resultado, nunca usó lápiz labial. Como profesionales dentales, no hay mayor satisfacción profesional o personal que la sonrisa en la cara de un paciente, especialmente si usted fue quien la diseñó especialmente para ese individuo.

Al utilizar materiales avanzados como el disilicato de litio para corregir problemas funcionales y estéticos, los dentistas y ceramistas pueden proporcionar a los pacientes resultados altamente funcionales y, lo que es más importante, una mayor confianza en sí mismos a través de la estética (Guess et al., 2010).

\section{REFERENCIAS BIBLIOGRÁFICAS}

1. Alex G. (2007). ¿Es realmente tan importante la oclusión y la odontología integral? Odontología interior.

2. Glosario de términos prostodónticos (1999). J Prosthet Dent.

3. Guess P, Zavanelli R, Silva N, et al. (2010). Disilicato de litio monolítico CAD / CAM versus coronas Y-TZP chapadas: comparación de modos de falla y confiabilidad después de la fatiga. Int J Prosthodont. 2

4. Höland W, Schweiger M, Frank M, et al. (2000). Una comparación de la microestructura y las propiedades de IPS Empress 2 y la cerámica de vidrio IPS Empress. J Biomed Mater Res.

5. Kheradmandan S, Koutayas S, Bernhard M, et al. (2001). Resistencia a la fractura de cuatro tipos diferentes de puentes anteriores de 3 unidades después de la fatiga termomecánica en el simulador de masticación de doble eje. J Rehabilitación oral.

6. Kinsel R, y Lin D. (2009). Análisis retrospectivo de fallas de porcelana de coronas de cerámica metálica y dentaduras postizas parciales fijas soportadas por 729 implantes en 152 pacientes: predictores de falla cerámica específicos del paciente y del implante. J Prosthet Dent. 
Revista Arbitrada Interdisciplinaria de Ciencias de la Salud. SALUD Y VIDA

Volumen 3. Número 6. Año 3. Julio - Diciembre 2019

Hecho el depósito de Ley: FA2016000010

ISSN: $2610-8038$

FUNDACIÓN KOINONIA (F.K)

Santa Ana de Coro, Venezuela.

Génesis Jesús Reyes Pico; Carlos Andres Pesantes Quijije; Triana Lucero Jimbo Bello

7. Mclntyre F. (2000). Restauración estética y guía anterior en dientes anteriores desgastados. Un enfoque multidisciplinario conservador. J. Am Dent Assoc.

8. Sorensen J, Cruz M, Mito W, et al. (1999). Una investigación clínica en prótesis parciales fijas de tres unidades fabricadas con una cerámica de vidrio de disilicato de litio. Pract Periodoncia Aesthet Dent.; cuestionario 108.

9. Spear F. (2007) Aproximaciones a la dimensión vertical. Odontología interior. 2007; 3: 34-43.

10. Wynne W. (2004). Consideraciones para establecer y mantener una oclusión adecuada en la zona estética. Dent Today.

\section{REFERENCES CONSULTED}

1. Alex G. (2007). Is occlusion and integral dentistry really so important? Interior dentistry

2. Glossary of prosthodontic terms (1999). J Prosthet Dent.

3. Guess P, Zavanelli R, Silva N, et al. (2010). CAD / CAM monolithic lithium disilicate versus Y-TZP plated crowns: comparison of failure modes and reliability after fatigue. Int J Prosthodont. 2

4. Höland W, Schweiger M, Frank M, et al. (2000). A comparison of the microstructure and properties of IPS Empress 2 and IPS Empress glass ceramics. J Biomed Mater Res.

5. Kheradmandan S, Koutayas S, Bernhard M, et al. (2001). Fracture resistance of four different types of anterior bridges of 3 units after thermomechanical fatigue in the dual-axis chewing simulator. J Oral rehabilitation.

6. Kinsel R, and Lin D. (2009). Retrospective analysis of porcelain faults of metal ceramic crowns and fixed partial dentures supported by 729 implants in 152 patients: specific predictors of patient and implant-specific ceramic failure. $\mathrm{J}$ Prosthet Dent.

7. Mclntyre F. (2000). Aesthetic restoration and anterior guide in worn anterior teeth. A conservative multidisciplinary approach. J. Am Dent Assoc. 
Revista Arbitrada Interdisciplinaria de Ciencias de la Salud. SALUD Y VIDA

Volumen 3. Número 6. Año 3. Julio - Diciembre 2019

Hecho el depósito de Ley: FA2016000010

ISSN: 2610-8038

FUNDACIÓN KOINONIA (F.K)

Santa Ana de Coro, Venezuela.

Génesis Jesús Reyes Pico; Carlos Andres Pesantes Quijije; Triana Lucero Jimbo Bello

8. Sorensen J, Cruz M, Mito W, et al. (1999). A clinical investigation in fixed partial prostheses of three units made with a lithium disilicate glass ceramic. Pract Periodontics Aesthet Dent .; questionnaire 108.

9. Spear F. (2007) Approaches to the vertical dimension. Interior dentistry 2007; 3: 34-43.

10. Wynne W. (2004). Considerations for establishing and maintaining adequate occlusion in the aesthetic zone. Dent Today.

(C2019 por los autores. Este artículo es de acceso abierto y distribuido según los términos y condiciones de la licencia Creative Commons Atribución-NoComercial-Compartirlgual 4.0 Internacional (CC BY-NC-

SA 4.0) (https://creativecommons.org/licenses/by-nc-sa/4.0/). 